\title{
The risk of small bowel adenocarcinoma in patients with Crohn's disease
}

\author{
Gustaw Lech ${ }^{1}$, Wojciech Korcz ${ }^{1}$, Emilia Kowalczyk², Andrzej Chaber ${ }^{1}$, Maciej Słodkowski ${ }^{1}$ \\ ${ }^{1}$ Department of General, Gastroenterological, and Oncological Surgery, Medical University of Warsaw, Warsaw, Poland \\ ${ }^{2}$ Student Science Group at Department of General, Gastroenterological, and Oncological Surgery, Medical University \\ of Warsaw, Warsaw, Poland
}

Gastroenterology Rev 2020; 15 (4): 309-313

DOI: https://doi.org/10.5114/pg.2020.101559

Key words: Crohn's disease, inflammatory bowel disease, small bowel adenocarcinoma, cancer, bowel.

Address for correspondence: Gustaw Lech MD, PhD, Department of General, Gastroenterological, and Oncological Surgery, Medical University of Warsaw, 1a Banacha St, 02-097 Warsaw, Poland, phone: +48 22599 24 82, fax: +48 2259920 57, e-mail: gustaw.lech@wum.edu.pl

\begin{abstract}
Introduction: Small bowel adenocarcinoma (SBA) is an unusual complication of Crohn's disease (CD).

Aim: To describe the incidence rate, clinical features, and outcomes of SBA in a group of subsequent patients with CD.

Material and methods: We retrospectively analysed outpatient and hospital records and identified a group of patients with diagnosed CD. Then we reviewed all medical records of patients who reported for follow-up visits in a 14-year period. We identified a group of 103 patients, whose medical records were evaluated for the presence of SBA.

Results: Long-term follow-up carried out in the group of consecutively treated patients with CD revealed an $0.97 \%$ incidence rate of SBA. Cancer was located in the ileum and the clinical presentation was subileus and anaemia. The patient underwent ileocaecal resection, and the postoperative period was uneventful.

Conclusions: SBA is a rare complication of CD. Although the authors are aware that the number of patients enrolled in the study is insufficient to draw far-reaching conclusions, the results obtained are significant for determination of the incidence rate of SBA in the Caucasian population of patients with CD. The key issue of effective treatment of patients with SBA was early detection of the lesion and RO resection with proper lymphadenectomy. However, it is worth noting that in more advanced stages of SBA the future belongs to, and outcome improvement depends on, new regimes of adjuvant personalised chemotherapies. Further studies on the mechanisms of carcinogenesis in patients with CD are essential.
\end{abstract}

\section{Introduction}

It is difficult to identify all factors increasing the risk of small bowel adenocarcinoma (SBA) due to the low prevalence of this cancer. The first case report with a demonstrated relationship between SBA and Crohn's disease (CD) was published by Ginzburg in 1956 [1]. It is a common belief that CD increases the risk of small bowel neoplasms, the most prevalent of which is SBA accounting for $57 \%$ of cases [2]. According to the literature, SBA develops in $0.08 \%$ to $5 \%$ of patients with CD [3-8]. A meta-analysis, published in 2010, of 20 papers from 1965 to 2008 based on 40,547 cases revealed that the incidence rate of SBA in patients with CD is 0.3 per 1000 patient-years (95\% Cl: 0.1-0.5/1000) [9]. The authors demonstrated a 18.75 -times higher risk of
SBA in patients with CD compared with a standard population from the same age group. Four meta-analyses published in the past [10-13] confirm a high relative risk for developing SBA, ranging from 17.4 to 28.37. Alternatively, its cumulative risk is $0.2 \%$ after 10 years and $2.2 \%$ after 25 years of the disease [14].

\section{Aim}

The aim of this paper was to describe the incidence rate, clinical features, and outcomes of small bowel adenocarcinoma in a group of subsequent patients with CD.

\section{Material and methods}

We retrospectively analysed the outpatient and hospital records and identified a group of patients with 
diagnosed CD treated in our department before 2003. The diagnosis of CD was established on either clinical, endoscopic, radiological, or histopathological evaluation. Then we retrospectively reviewed all the medical records of patients who reported for follow-up visits in our institution in a 14-year period of time (2003-2016). We identified a group of patients with diagnosed CD, whose medical records were evaluated for the presence of SBA. Patients with CD but whose medical records were incomplete were excluded. All patients included in the study met the criteria issued by the ECCO consensus recommendation for diagnosis and treatment [15]. The patients provided written informed consent. No approval was needed from the Local Ethics Committee because this was a retrospective study.

\section{Results}

The study group consisted of 103 Caucasian patients (56 female and 47 male) with mean age $49.3 \pm 12.8$ years (Table I). The mean CD duration in the study group was $19.0 \pm 4.6$ years. Patients' mean age at diagnosis was $29.0 \pm 9.1$ years. According to Montreal classification the assessed group consisted of 59 patients with disease location in terminal ileum (L1), 13 patients with Crohn's colitis (L2), 15 patients with multifocal CD affecting both ileum and large bowel (L3), and 16 patients with upper GI location of disease (L4). Our investigation revealed that CD behaviour evolved over the time and found $21.4 \%$ patients with penetrating type of disease (B3), $40.8 \%$ with stricturing disease (B2), and $37.8 \%$ with non-stricturing non-penetrating disease (B1). Pharmacological treatment in the investigated group of patients depended on disease activity and behaviour. 5-aminosalicylic acid agents (mesalazine and/ or less commonly sulphasalazine) were administered in $96.1 \%$ of patients. Steroid-dependent disease was considered in $18.4 \%$ of patients, among whom $89.4 \%$

Table I. Characteristics of the enrolled patients

\begin{tabular}{lc} 
Parameter & Value \\
\hline Number of patients & 103 (56 female, 47 male) \\
\hline Mean age at diagnosis [years] & $29.0 \pm 9.1$ \\
\hline Mean duration of disease [years] & $19.0 \pm 4.6$ \\
\hline Disease location & $\begin{array}{c}\text { Terminal ileum (L1) - 59, } \\
\text { large bowel (L2) }-13, \\
\text { multifocal (L3) }-15, \\
\text { upper Gl (L4) }-16\end{array}$ \\
\hline Disease behaviour & Non-stricturing \\
& $\begin{array}{r}\text { non-penetrating (B1) }-37.8 \%, \\
\text { stricturing (B2) }-40.8 \%, \\
\text { penetrating (B3) }-21.4 \%\end{array}$
\end{tabular}

were on oral prednisone or prednisolone and $10.6 \%$ were on budesonide. Azathioprine was used in $12.6 \%$ of patients. $9.7 \%$ of patients with objective evidence of active disease refractory to corticosteroids were treated with anti-TNF agents. Systematically acting antibiotics (metronidazole, ciprofloxacin) and anti-mycobacterial medications were administered occasionally as well. Despite intensive pharmacological treatment, the majority $(77.6 \%)$ of patients were treated surgically, and $28.7 \%$ among them were operated several times. Long-term follow-up (14 years) revealed 1 case of small bowel adenocarcinoma (0.97\%) out of 103 investigated patients with CD. SBA developed in a male patient (48 years old) with negative family history to either inflammatory bowel diseases or cancer. Crohn's disease duration at the time of SBA diagnosis was 6 years. Disease location was terminal ileum (L1) and it had stricturing behaviour (B2). The patient was previously treated chronically with amino salicylates during remission periods and periodically with oral glucocorticosteroids while the disease symptoms flared. He had not been operated before diagnosis. SBA was located in the ileum, and the clinical presentation comprised symptoms of subileus and anaemia: abdominal pain with bloating that exacerbated after meals, nausea, periodic vomiting, and significant fatigue. The patient underwent ileocaecal resection with primary anastomosis. Histopathology of a surgical specimen showed adenocarcinoma of the ileum G2 pT3NOMORO. The postoperative period was uneventful.

\section{Discussion}

Compared to de novo lesions, SBA secondary to CD develops in relatively young patients, and the median age at diagnosis ranges from 43.0 to 55.4 years $[2,3,6$, $7,16-24]$. In most patients, median duration of disease until cancer diagnosis is 12-24 years, but in 14-17\% of patients SBA develops significantly earlier, within 5 years of diagnosis of $C D[2,6,16-23]$. In the whole group of patients with $C D$, cancer is more likely to affect men (62.5-80\%) [20-23] and develops mostly in the ileum (73-89\%) and less frequently in the jejunum (11-25\%) [3, 6, 16, 18, 20-23]. In the examined group of patients with CD, the patient with SBA was typical in terms of the following: sex, age at diagnosis, location of tumour, and shortened duration of disease until cancer diagnosis. His medical history was negative regarding any factors predisposing to SBA: young age at diagnosis of $C D$, long-term remission, fistulas, bowel bypass, strictures or strictureplasty, and contact with chemical compounds.

The patients with $C D$, who were later diagnosed with SBA, presented with the following: abdominal 
pain (85.3-100\%), signs of Gl obstruction (30-89\%), weight loss (14.3-78\%), anaemia (28.5-50\%), diarrhoea $(20.5 \%)$, palpable tumour in the abdominal cavity (4.6-14.3\%), fever (14.3\%), GI bleeding (3.9-5.9\%), and bowel perforation (5.4\%) [6, 17, 18, 20, 21, 24]. Two highly pathognomonic clinical manifestations include recurrence of symptoms after a long-term remission and small bowel ileus resistant to treatment $[6,20]$. The patient described in our paper had recently complained of abdominal pain with bloating that exacerbated after meals, nausea, periodic vomiting, and significant fatigue. The previous long-term clinical history included periodic abdominal pain with diarrhoea and periodic nausea, iron-deficiency anaemia, skin lesions, arthralgia, and cholelithiasis.

Because it is difficult to distinguish these symptoms from Crohn's exacerbation, early diagnosis of cancer poses a significant challenge. These patients, often with a long-term history of inflammatory bowel disease, usually undergo endoscopy of the upper Gl tract and colon as a part of screening. When new symptoms develop or old ones exacerbate, initial imaging tests of the abdominal cavity are performed, i.e. an abdominal ultrasound and/or computer tomography (CT). A difficulty of imaging diagnostics of the small intestine in $\mathrm{CD}$ lies in differentiating small neoplastic lesions from severe CD. Contemporary diagnostic methods include advanced endoscopy and radiological techniques. Generally, if SBA is suspected in a patient with CD, detailed diagnostics begins with non-invasive radiological examinations. CT or magnetic resonance enterography is a good, frequently used, and safe diagnostic method that is well-tolerated by patients and achieves a sensitivity of above $70 \%$ [21]. Both tests may also be performed using enteroclysis; however, a nasoduodenal tube is not tolerated well by the majority of patients. Comparing the effectiveness of small bowel dilatation in both methods, greater intestinal distension was observed in enteroclysis, although it does not have a significant impact on the quality of intestinal assessment and accuracy of test results [25]. A pathological mass in imaging tests strongly suggests SBA in patients with $C D$; however, cancer may be completely indistinguishable from benign fibrosis or stricture secondary to acute inflammation $[6,17,18,21,26]$. In 1 case of SBA in the investigated group, a radiological $C T$ examination revealed a concentric ileal stricture $60 \mathrm{~mm}$ in length. If diagnosis cannot be made after radiological examinations, enteroscopy or video capsule endoscopy (VCE) may prove useful. VCE is the least invasive method of endoscopy, with a high sensitivity (5-67\%), allowing physicians to detect minor mucosal lesions that are not visualised in radiological examinations [27]. This meth- od often precedes enteroscopy, in particular in patients whose first symptom of SBA is GI bleeding. It should be used with great caution in patients with suspected mass in the small bowel and it is important to exclude subileus before VCE. Other diagnostic methods used in oncology, such as positron-emission tomography, have also become more available recently [28]. It was demonstrated that blood biochemistry and CEA levels are not useful indicators of both SBA development and its detection $[6,20]$. Due to diagnostic difficulties, SBA is detected incidentally (3.1-14\%) preoperatively and mostly postoperatively based on histopathology report. Lymph nodes are already involved in $33-55 \%$ of cases, and in $38-56 \%$ of cases metastases are observed $[6,8,17,18$, $20,24]$. Currently there is no standard management of SBA in patients with $C D$. The $R 0$ resection is the only approved radical treatment, and its area depends on disease severity and location of primary lesion. Surgery should result in a negative surgical margin and proper lymphadenectomy. Cancer of the jejunum and ileum should be resected with a margin of $5-10 \mathrm{~cm}$ of the macroscopically healthy intestine and corresponding mesentery up to superior mesenteric vessels in order to remove the appropriate amount of lymphatic tissue [26, 29-34]. Ileocaecal resection or right hemicolectomy should be performed if a tumour is located in the last ileal loop or has infiltrated the ileocaecal valve [5, 30-32, 35]. In the examined group of patients with $\mathrm{CD}$, the patient diagnosed with SBA was treated with ileocaecal resection. Planned radical resection is successful in $49-68 \%$ of surgeries, while palliative surgery is performed with a success rate of $12-35 \%$. Patients are mainly found ineligible for radical resection if their tumour has spread deeply to intestinal mesentery and distant organs [36]. The presence of distant metastases is a factor significantly increasing the incidence of postoperative complications and mortality. The overall survival (average 10 months) is comparable to non-operated patients. Despite this fact, as much as 30\% of patients with stage IV SBA are found to be eligible for palliative surgery, mainly due to heavy bleeding from the tumour site, or obstruction or perforation of the intestine [37]. Bowel bypasses and palliative resections are the most frequently performed palliative procedures $[30,32]$. Postoperative complications significantly decrease the quality of life and are observed in 14.5-60\% of patients [33, 36, 38, 39]. It was demonstrated that radical surgery has no significant impact on the number of postoperative complications. Total perioperative mortality ranges from $1 \%$ to $12 \%[36,39]$.

Currently available data on adjuvant chemotherapy in patients with SBA are very limited and provide discrepant information. In the investigated group, the 
patient diagnosed with SBA has not received adjuvant chemotherapy due to stage II cancer. It should be emphasised that only French guidelines (http://www.tncd. org) on adjuvant chemotherapy are available at present (updated in 2017). The authors recommend stage III cancer be treated with FOLFOX4 regimen (5-fluorouracil, oxaliplatin, folinic acid) for 6 months or, if tolerated poorly, with LV5FU2 regimen (5-fluorouracil, folinic acid) or capecitabine. Chemotherapy is not recommended in stage II cancer, except for T4 tumours when chemotherapy should be considered.

Optimistic information about a cytoreductive treatment in combination with hyperthermic intraperitoneal chemotherapy (HIPEC) in patients with colon cancer raises the hope that a similar outcome could be achieved in patients with SBA. The mean survival in one of the larger groups of 16 patients who underwent a cytoreductive treatment (CC-0 in $93.8 \%$ of them) and HIPEC was 30.8 months (3.4-94.4 months), and the time to recurrence amounted to 9.5 months (4.6-14.4 months) [40]. Intraperitoneal metastases were noted in 7 of 8 patients with recurrent disease. Indications for HIPEC should be considered each time in patients with intraperitoneal metastases due to prolonged survival observed in the discussed patients with SBA, along with acceptable toxicity of this treatment.

Data on the prognosis in patients with SBA secondary to $C D$ vary depending on the source, but it is commonly believed that it is poorer than in the case of de novo lesions. The 5-year survival rates are $3.7-35 \%$ and $14-45 \%$, respectively $[4,6,18-22]$. Mean survival is 13-40 months [5, 21, 26, 29, 32, 33, 35-39].

\section{Conclusions}

The long-term follow-up carried out in the group of consecutively treated patients with CD revealed a $0.97 \%$ incidence rate of small bowel adenocarcinoma. Although the authors are aware that the number of patients enrolled in the study is insufficient to draw far-reaching conclusions, the results obtained are significant for the determination of the incidence rate of SBA in the Caucasian population of patient with CD. The key issue of effective treatment of patient with SBA was in fact early detection of the lesion with typical symptoms and RO resection with proper lymphadenectomy. However, it is worth noting that in more advanced stages of SBA the future belongs to, and outcome improvement depends on, new regimes of adjuvant personalised chemotherapies. That is why further studies on the mechanisms of carcinogenesis in patients with CD are essential.

\section{Conflict of interest}

The authors declare no conflict of interest.

\section{References}

1. Ginzburg L, Schneider KM, Dreizin DH, et al. Carcinoma of the jejunum occurring in a case of regional enteritis. Surgery 1956; 39: 347-51.

2. Chang HK, Yu E, Kim J, et al. Adenocarcinoma of the small intestine: a multi-institutional study of 197 surgically resected cases. Hum Pathol 2010; 41: 1087-96.

3. Halfdanarson TR, McWilliams RR, Donohue JH, et al. A single-institution experience with 491 cases of small bowel adenocarcinoma. Am J Surg 2010; 199: 797-803.

4. Howe JR, Karnell LH, Menck HR, et al. The American College of Surgeons Commission on Cancer and the American Cancer Society. Adenocarcinoma of the small bowel: review of the National Cancer Data Base 1985-1995. Cancer 1999; 86: 2693-706.

5. Aparicio T, Zaanan A, Svrcek M, et al. Small bowel adenocarcinoma: epidemiology, risk factors, diagnosis and treatment. Dig Liver Dis 2014; 14: 97-104.

6. Cahill C, Gordon PH, Petrucci A, et al. Small bowel adenocarcinoma and Crohn's disease: any further ahead than 50 years ago? World J Gastroenterol 2014; 20: 11486-95.

7. Shaukat A, Virnig DJ, Howard D, et al. Crohn's disease and small bowel adenocarcinoma: a population-based case-control study. Cancer Epidemiol Biomarkers 2011; 20: 1120-3.

8. Tirkes AT, Duerinckx AJ. Adenocarcinoma of the ileum in Crohn's disease. Abdom Imaging 2005; 30: 671-3.

9. Laukoetter MG, Mennigen R, Hanning CM, et al. Intestinal cancer risk in Crohn's disease: a meta-analysis. J Gastrointest Surg 2011; 15: 576-83.

10. Jess T, Gamborg M, Matzen P, et al. Increased risk of intestinal cancer in Crohn's disease: a meta-analysis of population-based cohort studies. Am J Gastroenterol 2005; 100: 2724-9.

11. Canavan C, Abrams KR, Mayberry J. Meta-analysis: colorectal and small bowel cancer risk in patients with Crohn's disease. Aliment Pharmacol Ther 2006; 23: 1097-104.

12. von Roon AC, Reese G, Teare J, et al. The risk of cancer in patients with Crohn's disease. Dis Colon Rectum 2007; 50: 839-55.

13. Bernstein CN, Blanchard JF, Kliewer E, et al. Cancer risk in patients with inflammatory bowel disease: a population-based study. Cancer 2001; 91: 854-62.

14. Palascak-Juif V, Bouvier AM, Cosnes J, et al. Small bowel adenocarcinoma in patients with Crohn's disease compared with small bowel adenocarcinoma de novo. Inflamm Bowel Dis 2005; 11: 828-32.

15. Van Assche G, Dignass A, Panes J, et al. The second European evidence-based Consensus on the diagnosis and management of Crohn's disease: definitions and diagnosis. J Crohns Colitis 2010; 4: 7-27.

16. Feldstein RC, Sood S, Katz S. Small bowel adenocarcinoma in Crohn's disease. Inflamm Bowel Dis 2008; 14: 1154-7.

17. Solem CA, Harmsen WC, Zinsmeister AL, et al. Small intestinal adenocarcinoma in Crohn's disease. A case-control study. Inflamm Bowel Dis 2004; 10: 32-5.

18. Weber NK, Fletcher JG, Fidler JL, et al. Clinical characteristics and imaging features of small bowel adenocarcinomas in Crohn's disease. Abdom Imaging 2015; 40: 1060-7. 
19. Christodoulou D, Skopelitou AS, Katsanos KH, et al. Small bowel adenocarcinoma presenting as a first manifestation of Crohn's disease: report of a case, and a literature review. Eur J Gastroenterol Hepatol 2002; 14: 805-10.

20. Dossett LA, White LM, Welch DC, et al. Small bowel adenocarcinoma complicating Crohn's disease: case series and review of the literature. Am Surg 2007; 73: 1181-7.

21. Soyer P, Hristova L, Boudghene F, et al. Small bowel adenocarcinoma in Crohn disease: CT-enterography features with pathological correlation. Abdom Imaging 2012; 37: 338-49.

22. Menon AM, Mirza AH, Moolla S, et al. Adenocarcinoma of the small bowel arising from a previous strictureplasty for Crohn's disease: report of a case. Dis Colon Rectum 2006; 50: 257-9.

23. Whitcomb E, Liu X, Xiao SY. Crohn enteritis-associated small bowel adenocarcinomas exhibit gastric differentiation. Human Pathol 2014; 45: 359-67.

24. Placé V, Hristova L, Dray X, et al. Ileal adenocarcinoma in Crohn's disease: magnetic resonance enterography features. Clin Imaging 2012; 36: 24-8.

25. Negaard A, Sandvik L, Berstad AE, et al. MRI of the small bowe with oral contrast or nasojejunal intubation in Crohn's dis ease: randomized comparison of patient acceptance. Scand J Gastroenterol 2008; 43: 44-51.

26. Ugurlu MM, Asoglu O, Potter DD, et al. Adenocarcinomas of the jejunum and ileum: a 25-year experience. J Gastrointest Surg 2005; 9: 1182-8.

27. Eliakim R. Wireless capsule video endoscopy: three years of experience. World J Gastroenterol 2004; 10: 1238-9.

28. Cronin CG, Scott J, Kambadakone A, et al. Utility of positron emission tomography/CT in the evaluation of small bowel pathology. Br J Radiol 2012; 85: 1211-21.

29. El Zouhairi M, Venner A, Charabaty A, et al. Small bowel adenocarcinoma. Curr Treat Options Oncol 2008; 9: 388-99.

30. Reynolds I, Healy P, Mcnamara DA. Malignant tumours of the small intestine. Surgeon 2014; 12: 263-70.

31. Guo X, Mao Z, Su D, et al. The clinical pathological features, diagnosis, treatment and prognosis of small intestine primary malignant tumors. Med Oncol 2014; 31: 913-7.

32. Wu TJ, Yeh CN, Chao TC, et al. Prognostic factors of primary small bowel adenocarcinoma: univariate and multivariate analysis. World J Surg 2006; 30: 391-8.

33. Chaiyasate K, Jain AK, Cheung LY, et al. Prognostic factors in primary adenocarcinoma of the small intestine: 13-year single institution experience. World J Surg Oncol 2008; 6: 12-8.

34. Neely D, Ong J, Patterson J, et al. Small intestinal adenocarcinoma: rarely considered, often missed? Postgrad Med J 2013; 89: 197-201.

35. Dabaja BS, Suki D, Pro B, et al. Adenocarcinoma of the small bowel: presentation, prognostic factors, and outcome of 217 patients. Cancer 2004; 101: 518-26.

36. Agrawal S, McCarron EC, Gibbs JF, et al. Surgical management and outcome in primary adenocarcinoma of the small bowel. Ann Surg Oncol 2007; 14: 2263-9.

37. Hong SH, Koh YH, Rho SY, et al. Primary adenocarcinoma of the small intestine: presentation, prognostic factors and clinical outcome. Jpn J Clin Oncol 2009; 39: 54-61.

38. Schwameis K, Schoppmann SF, Stift J, et al. Small bowel adenocarcinoma - terra incognita: a demand for cross-national pooling of data. Oncol Lett 2014; 7: 1613-7.
39. Goodman MT, Matsuno RK, Shvetsov YB. Racial and ethnic variation in the incidence of small-bowel cancer subtypes in the United States, 1995-2008. Dis Colon Rectum 2013; 56: 441-8.

40. van Oudheusden TR, Lemmens VE, Braam HJ, et al. Peritoneal metastases from small bowel cancer: results of cytoreductive surgery and hyperthermic intraperitoneal chemotherapy in The Netherlands. Surgery 2015; 157: 1023-7.

Received: 18.09 .2019

Accepted: 4.11 .2019 